\title{
Capital psicológico y regulación emocional en el trabajo: El rol mediador de la satisfacción laboral
}

Solana Salessi. Universidad Nacional de Rafaela. Pontificia Universidad Católica Argentina. Alicia Omar. Consejo Nacional de Investigaciones Científicas y Técnicas.

\section{Resumen}

El objetivo del presente artículo fue analizar el impacto de la satisfacción laboral sobre las relaciones entre el capital psicológico de los trabajadores y las estrategias que emplean para regular sus emociones. La verificación empírica se realizó sobre una muestra de 386 trabajadores de empresas de servicios argentinas. Se plantearon diferentes hipótesis sobre las relaciones directas e indirectas entre las variables estudiadas, las que fueron probadas mediante análisis de correlación y de ecuaciones estructurales. Los resultados mostraron que la satisfacción laboral se desempeña como mediador total. Es decir que la totalidad de la varianza de la relación entre el capital psicológico y las estrategias de regulación emocional depende de la satisfacción de los trabajadores. Se discuten los resultados obtenidos y se señalan las fortalezas y limitaciones del estudio realizado. Se sugieren lineamientos para futuros estudios.

Palabras clave: regulación emocional; satisfacción en el trabajo; capital psicológico; psicología organizacional.

\section{Resumo}

Capital psicológico e regulação emocional no trabalho: O papel mediador da satisfação no trabalho. O objetivo deste trabalho foi analisar o impacto da satisfação no trabalho sobre a relação entre capital psicológico dos trabalhadores e as estratégias que eles usam para regular as emoções. A verificação empírica foi realizada em uma amostra de 386 trabalhadores argentinos de empresas de serviços. Diferentes hipóteses sobre as relações diretas e indiretas entre as variáveis estudadas foram levantadas, as que foram testadas por análise de correlação e de equações estruturais. Os resultados mostraram que a satisfação no trabalho funciona como um mediador completo. Isso significa que toda a variação da relação entre o capital psicológico e estratégias de regulação emocionais depende da satisfação dos trabalhadores. Tais resultados são discutidos. Os pontos fortes e limitações do estudo são identificados. Diretrizes para futuros estudos são sugeridos.

Palavras chave: regulação emocional; satisfação no trabalho; capital psicológico; psicologia organizacional.

\begin{abstract}
Psychological capital and emotional regulation: The mediating role of job satisfaction. The aim of this paper was to analyze the impact of job satisfaction on the relationship between psychological capital and the workers strategies to regulate emotions are analyzed. The empirical verification was performed on a sample of 386 employees of Argentinean companies of services. Different hypotheses about the direct and indirect relationships between the variables studied were postulated, which were tested by correlation analysis and structural equation modeling. The results showed that job satisfaction serves as a full mediator. This means that the entirety of the variance of the relationship between psychological capital and emotional regulation strategies depends on the satisfaction of workers. The results are discussed and the strengths and limitations of the study are indicated. Guidelines for future studies are suggested.
\end{abstract}

Keywords: emotional regulation; job satisfaction; psychological capital; organizational psychology. 
El estudio de la regulación emocional en el trabajo ha crecido de manera constante y sostenida, alcanzado su posición más destacada durante la última década (Grandey, 2015). El constructo regulación emocional hace referencia al conjunto de procesos mediante los cuales los sujetos ejercen influencia sobre las emociones que experimentan, y sobre el modo cómo las expresan (Gross, 2010). Entre las diversas tipologías disponibles, la propuesta por Hochschild (1983) se ha impuesto como el marco teórico de referencia para analizar la regulación emocional en el ámbito laboral. En este sentido, la actuación profunda y la actuación superficial constituyen las principales estrategias en torno a las cuales se ha centrado la literatura organizacional (Grandey, Diefendorff, \& Rupp, 2013).

En la actualidad, los estudiosos de la temática se encuentran abocados a desentrañar los mecanismos que subyacen al uso de cada estrategia enfatizando, especialmente, la identificación de las variables que pueden actuar como antecedentes o facilitadores. La evidencia reunida hasta el momento muestra que, además de los factores contextuales o situacionales tradicionalmente considerados, las diferencias individuales también podrían explicar porciones significativas de su varianza (Liu, Prati, Perrewe, \& Brymer, 2010). Si bien la personalidad ha sido una de las candidatas predilectas de los investigadores, la tendencia vigente se orienta a explorar el potencial explicativo de otras variables disposicionales. Tal es el caso del capital psicológico o CapPsi (Luthans, Youssef, \& Avolio, 2007). En este sentido, investigaciones muy recientes (Begenirbaş, 2015; Hur, Rhee, \& Ahn, 2016; Uğurlu-Kara \& Begenirbaş, 2016) han sugerido posibles vinculaciones entre los recursos psicológicos de los empleados y las estrategias de regulación emocional que éstos implementan. Asimismo, se ha postulado (Mao, \& Tang, 2015; Wei, Guo, Ye, Liao, \& Yang, 2016) que variables actitudinales, tales como la satisfacción laboral, podrían intervenir como mecanismos explicativos en tales relaciones.

Como un indicador del grado de avance y madurez que está alcanzado la disciplina, la psicología organizacional contemporánea ya no se circunscribe a analizar relaciones directas, desarrollando cada vez más investigaciones de segunda generación (Hopwood, 2007). Es decir, estudios orientados a identificar terceras variables que medien o moderen el comportamiento de otras; influyendo así en la intensidad y dirección de sus vinculaciones. El presente trabajo se encuadró, precisamente, en esta segunda perspectiva, desde el momento que su objetivo fue examinar si la satisfacción laboral emergía como un engranaje clave en las vinculaciones entre el CapPsi y la regulación emocional. Vale decir, explorar si la satisfacción laboral desempeña el rol de mediador, explicando total o parcialmente las relaciones entre los recursos psicológicos y las estrategias emocionales implementadas por los trabajadores.

\section{Regulación Emocional}

Los esfuerzos de regulación pueden estar dirigidos a modificar los sentimientos de base o la forma en que las emociones se manifiestan. En este sentido, la actuación superficial constituye una estrategia circunscripta únicamente a los aspectos explícitos de la respuesta emocional. Por lo que se caracteriza por la manipulación de las expresiones faciales y el lenguaje corporal, en un intento por fingir una emoción o evitar ser delatados por ella. La actuación profunda, en cambio, se dirige a modificar los aspectos subjetivos y experienciales, siendo su propósito fundamental evocar una emoción genuina compatible con los requerimientos organizacionales o las expectativas del interlocutor (Grandey et al., 2013; Hochschild, 1983). A la luz de las categorías planteadas por Gross (2010), la actuación profunda puede ser considerada una estrategia centrada en los antecedentes, habida cuenta que pretende influir en el proceso generativo de la emoción. Contrariamente, la actuación superficial constituye una estrategia focalizada en la respuesta, ya que se ciñe al montaje de una suerte de "pantalla" socialmente aceptable, con el único propósito de adecuarse a los lineamientos organizacionales (Grandey, 2015).

\section{Regulación Emocional y Capital Psicológico}

El CapPsi (Luthans et al., 2007) constituye un estado psicológico caracterizado por la confianza para asumir una tarea desafiante, efectuar atribuciones positivas acerca del resultado de la misma, perseverar en la búsqueda de los objetivos y, salir fortalecido de la adversidad. Las fortalezas psicológicas que integran el constructo (esperanza, auto-eficacia, optimismo y resiliencia) se traducen en mayor bienestar y calidad de vida para los trabajadores, al tiempo que potencian la rentabilidad y las ventajas competitivas de las organizaciones (Omar, 2015).

Investigaciones recientes han demostrado relaciones modestas pero estadísticamente significativas entre los recursos que integran el CapPsi y las estrategias de regulación emocional. Por ejemplo, analizando 
una muestra de profesionales de hospitales públicos y privados turcos, Begenirbaş (2015) ha observado que cuando la autoeficacia, la esperanza y el optimismo son altos, la actuación superficial de los empleados y la alienación con el trabajo son menores, al tiempo que son elevadas las demostraciones emocionales genuinas. En línea con tales resultados, un estudio coreano realizado sobre una muestra de 263 azafatas (Hur et al., 2016), encontró relaciones positivas entre el suprafactor CapPsi y la actuación profunda. Paralelamente, se han comunicado (Uğurlu-Kara \& Begenirbaş, 2016) relaciones negativas entre el CapPsi y la actuación superficial entre empleados de hotel. Tales hallazgos pueden ser comprendidos a partir de la teoría de conservación de recursos (Hobfoll, 2011). Uno de sus postulados centrales señala que las personas se esfuerzan por obtener, acumular y proteger sus recursos a la hora de afrontar demandas y presiones; las que, en este caso, estarían representadas por los requerimientos organizacionales o profesionales en torno a la regulación y el autocontrol emocional. Desde esta perspectiva, resulta congruente que los trabajadores que poseen elevado CapPsi procuren resguardar ese estado positivo. De allí que, a la hora de cumplir determinadas reglas (implícitas o explícitas) en materia de expresión emocional, estos empleados traten de responder con estrategias capaces de salvaguardar sus recursos e, incluso, generar nuevos. Contrariamente, los empleados con menor CapPsi tratarían de no invertir su exiguo capital en estrategias que requieran altos niveles de compromiso y esfuerzo, circunscribiéndose simplemente a controlar las "válvulas de escape" de sus emociones (es decir, las expresiones faciales, las miradas, los gestos, la cadencia y el tono de voz, etc.). En línea con tales conjeturas, se ha señalado (Grandey et al., 2013) que la actuación superficial aún siendo una estrategia más sencilla y acotada tiene marcados efectos nocivos, desde el momento que no permite generar nuevos recursos y agota los movilizados para su ejecución. La actuación profunda, en cambio, es la única capaz de proporcionar el acceso a otros "activos", tales como confianza, autoestima, realización personal, emociones positivas, desarrollo de redes sociales, etc. Con base en estos referentes empíricos y teóricos se hipotetiza que:

Hipótesis 1: el CapPsi se relacionará negativamente con la actuación superficial.

Hipótesis 2: el CapPsi se relacionará positivamente con la actuación profunda.

\section{Regulación Emocional, Capital Psicológico y Satisfacción Laboral}

Desde su irrupción en el ámbito académico, la satisfacción laboral ha sido objeto de diversas conceptualizaciones. Estas incluyen desde su consideración como un sentimiento positivo, a su definición en términos de la favorabilidad (o desfavorabilidad) con la que los empleados evalúan su trabajo (Salessi, 2014). En los últimos años, ha comenzado a cobrar fuerza la alternativa de concebirla como un conjunto de respuestas cognitivas y afectivas a la situación laboral, con importantes implicancias en el comportamiento de los empleados y en los resultados organizacionales (Judge, \& KammeyerMueller, 2012). En este sentido, los trabajadores satisfechos suelen experimentar mayor felicidad y bienestar; en tanto que las organizaciones pueden verse recompensadas por ejecuciones destacadas, que se traducen en aumentos de la productividad, mayor compromiso y menores intenciones de renunciar por parte de sus miembros (Paris \& Omar, 2008).

A la fecha se dispone de cierta evidencia acerca de las relaciones directas entre el CapPsi y la satisfacción laboral. Al respecto, un reciente estudio longitudinal (Alessandri, Borgogni, Consiglio, \& Mitidieri, 2015) ejecutado sobre una muestra de 465 trabajadores de una empresa italiana demostró que el CapPsi predice significativamente la satisfacción y el compromiso de los trabajadores. Paralelamente, una investigación realizada sobre 451 empleados representantes de los diversos sectores de la economía egipcia (Badran \& Youssef-Morgan, 2015) informó relaciones positivas entre CapPsi y satisfacción laboral. A similares conclusiones han arribado Salessi y Omar (2016b) a partir de su estudio con una muestra multi-ocupacional de trabajadores argentinos. En conjunto, tales resultados no hacen sino ratificar los hallazgos previamente comunicados por Avey, Reichard, Luthans y Mhatre (2011) al dejar firmemente establecido que el CapPsi se asocia positivamente a la satisfacción de los trabajadores en un grado comparablemente superior al de cualquiera de sus componentes individuales.

En lo que hace a las vinculaciones entre la satisfacción laboral y la regulación emocional, la literatura muestra que, mientras las actuaciones profundas se traducen en mayores niveles de satisfacción, las actuaciones superficiales contribuyen a su detrimento (Jiang, Jiang, \& Park, 2013; Salessi \& Omar, 2016a). Sin embargo, se desconoce aún si la satisfacción puede influir en la implementación de una u otra estrategia. Al respecto, diversos estudios 
(Mao, \& Tang, 2015; Wei et al., 2016) vienen demostrando que la satisfacción laboral actúa como mediador en las relaciones entre antecedentes disposicionales (tales como el CapPsi) y manifestaciones comportamentales en el lugar de trabajo (en este caso, las estrategias de regulación emocional). En sintonía con tales evidencias empíricas, el marco conceptual provisto por el modelo de ampliación y construcción emocional (Fredrickson, 2001) permite comprender más acabadamente el posible rol mediador de la satisfacción. De acuerdo con esta perspectiva teórica, los estados emocionales positivos incrementan los recursos psicológicos y amplifican las posibilidades de la atención, la cognición y la acción, traduciéndose esto en una espiral ascendente de emocionalidad positiva. La literatura internacional (Judge \& Kammeyer-Mueller, 2012; Salessi \& Omar 2016b) coincide en resaltar que la satisfacción se asocia intrínsecamente a un estado emocional placentero y, como tal, a un factor motivacional positivo con efecto significativo sobre el comportamiento. Por lo tanto, el componente afectivo que la satisfacción conlleva podría potenciar los recursos psicológicos así como originar un estado de sincronización emocional frente a la necesidad de demostrar emociones positivas. De esta manera, respuestas emocionales auténticas y comprometidas como las que supone la actuación profunda podrían verse facilitadas ante elevados niveles de CapPsi. Por el contrario, el descontento asociado a la insatisfacción podría consolidar la exigua voluntad que caracteriza a los trabajadores con menos CapPsi, además de suscitar respuestas apáticas y desapegadas como las que implica la actuación superficial. Con base en estos antecedentes se conjetura que:

Hipótesis 3: la satisfacción laboral mediará las relaciones entre el CapPsi y las estrategias de actuación profunda y actuación superficial.

\section{Método}

\section{Diseño}

La presente investigación corresponde a un estudio empírico, cuantitativo y transversal. Se enmarca en los lineamientos de la estrategia asociativa-explicativa (Ato, López, \& Benavente, 2013), ya que su finalidad fue explorar la relación funcional existente entre variables.

\section{Participantes}

Se trabajó con una muestra por conveniencia (no probabilística), siendo el criterio de inclusión que los participantes trabajaran en organizaciones comerciales y de servicios. Dicho criterio obedeció a que en estas ocupaciones, dado el contacto permanente con clientes, la regulación de las emociones constituye una tarea inherente al rol. Por lo que la manifestación de actuaciones profundas y/o superficiales se observa en mayor medida que en otras profesiones con menores requerimientos interpersonales (Salessi, 2012).

Siguiendo las recomendaciones de Tabachnick y Fidell (2013) de contar con un mínimo de 200 casos para garantizar la verificación de los supuestos del análisis de ecuaciones estructurales, la muestra definitiva fue de 386 personas. Esta muestra, que superó ampliamente el tamaño muestral mínimo sugerido por los especialistas, quedó integrada por 168 varones y 218 mujeres, miembros de organizaciones públicas y privadas argentinas radicadas en las provincias de Buenos Aires y de Santa Fe. La edad promedio de los participantes fue de 34 años (DT $=9.65$ ) y la antigüedad laboral media de 5 años (DT = 7.48). El 54\% de los participantes tenían estudios superiores (terciarios o universitarios), y en cuanto al rubro de actividad de sus empresas, el $41 \%$ pertenecía al comercio, el $34 \%$ a la hotelería y el $25 \%$ a la administración pública.

\section{Procedimiento}

La recolección de los datos se llevó a cabo durante el segundo semestre del año 2015. Todos los participantes fueron contactados en sus respectivos lugares de trabajo y, luego de informales sobre los objetivos del estudio, se los invitó a responder un cuadernillo integrado por las escalas de Capital Psicológico, de Regulación Emocional y de Satisfacción Laboral, previa firma de un protocolo de consentimiento. La participación fue voluntaria, anónima y confidencial. No se brindaron incentivos de ningún tipo. La administración de las técnicas estuvo a cargo de personal entrenado a tal efecto. La investigación se realizó en un todo de acuerdo con los lineamientos éticos recomendados tanto por la American Psychological Association (APA) como por la Comisión para las Ciencias Sociales y Humanas del CONICET (Resolución 2827/06).

\section{Instrumentos}

Capital psicológico. Se midió con la escala homónima desarrollada por Omar, Salessi y Urteaga (2014). El instrumento está integrado por 16 ítems con formato de respuesta tipo Likert de 5 puntos (variando de 1= nunca a $5=$ siempre), que miden las cuatro facetas del CapPsi a razón de 4 ítems por escala: esperanza (ej.: "tengo 
fe de que al fin mis asuntos laborales van a mejorar"); optimismo (ej.: "miro el lado positivo de cada proyecto laboral que emprendo"); resiliencia (ej.: "cuando tengo una dificultad en mi trabajo, la supero con éxito"), y autoeficacia (ej.: "si me esfuerzo lo necesario, puedo resolver problemas difíciles en mi trabajo"

Estrategias de actuación. Se midieron con la escala de Estrategias de Actuación Emocional en el Trabajo (Salessi, \& Omar, 2016a). La misma consta de seis ítems con formato de respuesta tipo Likert de 5 puntos, variando entre 1 (nunca) y 5 (siempre). Tres reactivos exploran actuación superficial (ej.: "en mi trabajo soy encantador sólo para causar una buena impresión"), en tanto que los tres restantes miden actuación profunda (ej.: "cuando me siento mal en mi trabajo, trato de ver las cosas desde otro punto de vista").

Satisfacción laboral. Fue evaluada mediante la adaptación argentina (Salessi, \& Omar, 2016b) de la Escala de Satisfacción Laboral Genérica. El instrumento está integrado por siete ítems (ej.: "en mi trabajo puedo aplicar todas mis capacidades y habilidades"), y proporciona una estimación global del grado de satisfacción laboral. Cada ítem es valorado sobre una escala tipo Likert de 5 puntos, que va desde 1 (totalmente en desacuerdo) hasta 5 (totalmente de acuerdo).

\section{Estrategia de Análisis de los Datos}

Se examinó la matriz de datos con miras a detectar valores perdidos, y se analizó la distribución conjunta de las variables a través del cómputo del coeficiente de curtosis multivariada estandarizado (Bentler, 2006). Se ejecutó un análisis factorial confirmatorio (AFC) para determinar el ajuste del modelo de medida utilizado. Vale decir, un modelo conformado por los factores satisfacción laboral, actuación profunda, actuación superficial y CapPsi (modelado como un factor de segundo orden); sus respectivos ítems como indicadores observables y sus respectivos términos de error. La confiabilidad se examinó a partir del coeficiente alfa de Cronbach y del índice de confiabilidad compuesta (CR, Composite Reliability). La validez convergente, se determinó a través de la varianza media extraída (AVE, Average Variance Extracted). La validez discriminate se analizó con base en la raíz cuadrada del AVE. La presencia de potenciales sesgos debidos al método común se evaluó a partir de la prueba de un solo factor de Harman (Podsakoff, MacKenzie, \& Podsakoff, 2012). Se calcularon los índices descriptivos (medias y desvíos típicos). Se realizaron pruebas de diferencias de medias ( $t$ de Student) para el género, y análisis de varianza (ANOVA de un factor) para el nivel de estudios y la actividad. Tal procedimiento se realizó a fin de detectar posibles diferencias significativas que pudieran ameritar incluir a estas características sociodemográficas como variables control.

La verificación empírica de las hipótesis propuestas se realizó a partir de su modelización con ecuaciones estructurales. En línea con el procedimiento clásico (Baron \& Kenny, 1986), en primer lugar se examinaron las premisas que rigen el mecanismo de medicación, a saber: (a) que la variable independiente (CapPsi) se relacione con las variables dependientes (actuación profunda y actuación superficial); (b) que la variable independiente se relacione con el potencial mediador (satisfacción laboral) y que; (c) la variable mediadora se relacione con las variables dependientes. Para determinar el tipo de mediación, vale decir, toral o parcial, se optó por la estrategia de modelos rivales (Bentler, 2006), contrastando un modelo de efectos indirectos o de mediación total (modelo A) y un modelo de efectos indirectos y directos o de mediación parcial (modelo B). Para la estimación de los modelos se empleó el método de máxima verosimilitud con la corrección robusta de Satorra-Bentler (S-B), recomendado cuando los datos provienen de escalas tipo Likert (Bentler, 2006). Para evaluar la bondad de ajuste se analizó que la razón entre índice chi-cuadrado corregido no fuera significativo y los grados de libertad $\left(\mathrm{S}-\mathrm{BX}^{2} / g \mathrm{gl}\right)$ fuera inferior a 4; que el índice de bondad de ajuste (GFI, Goodness of Fit Index) y el índice de ajuste comparativo (CFI, Comparative Fit Index) alcanzaran valores iguales o superiores a .90 , y que el valor del error cuadrático medio de aproximación (RMSEA, Root Mean Square Error of Approximation) fuera inferior a .05. Adicionalmente, se analizó el índice esperado de validación cruzada (ECVI, Expected Cross-Validation Index) y el criterio consistente de información de Akaike (CAIC, Consistent Akaike Information Criterion), considerando que el modelo más adecuado sería el que presente valores comparativamente más pequeños. Complementariamente, se computó la prueba de diferencias entre los estadísticos chi-cuadrado $\left(\mathrm{S}-\mathrm{B} \chi^{2}\right)$ y se ejecutó un análisis con bootstrapping para determinar la significación estadística de los efectos directos e indirectos. Con tal propósito, se seleccionaron 5.000 muestras al azar del conjunto de datos y se estimaron los intervalos de confianza al 95\%. Los análisis fueron ejecutados con los softwares SPSS (versión 19.0) y EQS (versión 6.0). 


\section{Resultados}

\section{Análisis Preliminares}

El coeficiente de curtosis multivariante estandarizado fue de 5.21 encontrándose fuera del intervalo $(-3 ; 3)$ recomendado para asumir multinormalidad (Bentler, 2006). Tal resultado ameritó el uso de estimadores robustos para el cálculo de los parámetros, tanto del modelo de medida como del modelo estructural. El modelo de medida conformado por cuatro variables latentes correlacionadas, sus respectivos ítems como indicadores observables y los correspondientes errores de medida, se ajustó adecuadamente a los datos empíricos $\left(\mathrm{S}-\mathrm{B} \chi^{2} / g l=2.96 ; \mathrm{GFI}=.98\right.$; $\mathrm{CFI}=.99$; RMSEA $=.02)$. Los ítems presentaron cargas factoriales entre .76 y $.98(p<.001)$ en sus respectivos factores. Para todas las variables, la confiabilidad compuesta superó el mínimo de .70, el índice AVE para cada constructo fue mayor a .50, y la raíz cuadrada del mismo fue superior que la correlación entre ese constructo y todos los demás (Fornell \& Larcker, 1981). La prueba de Harman indicó que la varianza explicada por un solo factor era del $18.42 \%$, lo que evidenció que los datos no se encontraban sesgados.

\section{Verificación Empírica}

En la Tabla 1 se presentan los estadísticos descriptivos, los indicadores de confiabilidad, y los índices de validez convergente y discriminante.

Ni las pruebas $t$ de Student ni los análisis de la varianza arrojaron resultados significativos. La falta de diferencias atribuibles a las variables sociodemográficas (definidas en el presente estudio como variables control), ameritaron que no fueran incluidas en los análisis posteriores.

El examen de los coeficientes correspondientes a las tres relaciones analizadas demostró el cumplimiento de las premisas de la mediación. En este sentido, se encontró que el CapPsi se relacionó positivamente con la actuación profunda $(\beta=0,24 ; p<0,01)$ y negativamente con la actuación superficial $(\beta=-0,20 ; p<0,01)$. Estos resultados brindan apoyo empírico a las hipótesis 1 y 2. Asimismo, el CapPsi se vinculó positivamente con la satisfacción laboral $(\beta=0,35 ; p<0,01)$, en tanto que ésta se asoció positivamente a la actuación profunda $(\beta=0,48 ; p<0,01)$ y negativamente a la actuación superficial $(\beta=-0,60 ; p<0,01)$.

El análisis de los índices de ajuste indicó que el modelo de mediación total presenta un ajuste más satisfactorio a los datos empíricos (S-BX ${ }^{2}=9,68 ; \mathrm{S}-\mathrm{BX}^{2} / \mathrm{gl}=3,22 ; \mathrm{GFI}$ $=0,99 ; \mathrm{CFI}=0,98 ; \mathrm{RMSEA}=0,02 ; \mathrm{ECVI}=0,42 ; \mathrm{CAIC}=27,89$ ), en comparación al modelo de mediación parcial $\left(\mathrm{S}-\mathrm{BX}^{2}=\right.$ 5,60; $\mathrm{S}-\mathrm{BX}^{2} / \mathrm{gI}=5,60 ; \mathrm{GFI}=0,95 ; \mathrm{CFI}=0,93 ; \mathrm{RMSEA}=0,07$; $\mathrm{ECVI}=0,63$; CAIC $=62,46)$. A su vez, la prueba de diferencias entre los $\mathrm{S}-\mathrm{B} \chi^{2}$ resultó no significativa $\left(\mathrm{S}-\mathrm{B} \mathrm{X}^{2}=4.08 ; \mathrm{gl}=\right.$ $2 ; p=.130$ ), demostrando que no existen diferencias entre los modelos A y B. Vale decir que, la inclusión de efectos directos residuales por parte del CapPsi en el modelo de mediación parcial no supone ninguna mejora respecto del modelo de mediación total en el que tal relación no es considerada. Estos resultados fueron ratificados por el análisis de bootstrapping (Tabla 2). De este modo, la hipótesis 3 queda empíricamente confirmada.

La Figura 1 resume los coeficientes estandarizados y proporciones de varianzas explicadas para las variables endógenas.

Tabla 1. Estadísticos Descriptivos e Índices de Confiabilidad y Validez Correspondientes a las Variables en Estudio.

\begin{tabular}{lcccccc}
\hline & $\mathbf{X}$ & DT & $\boldsymbol{a}$ & CR & AVE & Raíz cuadrada AVE \\
\hline 1. CapPsi & 4,06 & 0,42 & 0,89 & 0,88 & 0,72 & 0,84 \\
2. Actuación Profunda & 3,59 & 0,71 & 0,88 & 0,80 & 0,65 & 0,81 \\
3. Actuación Superficial & 2,41 & 0,94 & 0,90 & 0,87 & 0,73 & 0,76 \\
4. Satisfacción Laboral & 3,52 & 0,85 & 0,87 & 0,90 & & 0,87 \\
5. Edad & 34,07 & 9,65 & & & \\
6. Antigüedad Laboral & 5,01 & 7,78 & & & \\
\hline
\end{tabular}

Tabla 2. Coeficientes Estandarizados y Intervalos de Confianza Correspondientes a los Efectos Indirectos y Directos Del Cappsi sobre las Estrategias de Regulación Emocional.

\begin{tabular}{lcccc}
\hline \multirow{2}{*}{ Variable dependiente } & \multicolumn{2}{c}{$\begin{array}{c}\text { Efecto indirecto del CapPsi } \\
\text { (con mediación de la satisfacción laboral) }\end{array}$} & \multicolumn{2}{c}{$\begin{array}{c}\text { Efecto directo residual del CapPsi } \\
\text { (sin mediación de la satisfacción laboral) }\end{array}$} \\
\cline { 2 - 5 } & Coef. estadarizado & IC al 95\% & Coef. estadarizado & IC al 95\% \\
\hline Actuación Profunda & $0,17^{* *}$ & 0,$09 ; 0,23$ & $0,01^{\text {ns }}$ & $-0,05 ; 0,09$ \\
Actuación Superficial & $-0,21^{* *}$ & $-0,28 ;-0,19$ & $-0,04^{\text {ns }}$ & $-0,07 ; 0,08$ \\
\hline
\end{tabular}

${ }^{* *}=\mathrm{p}<0,001 ; \mathrm{ns}=$ no significativo 


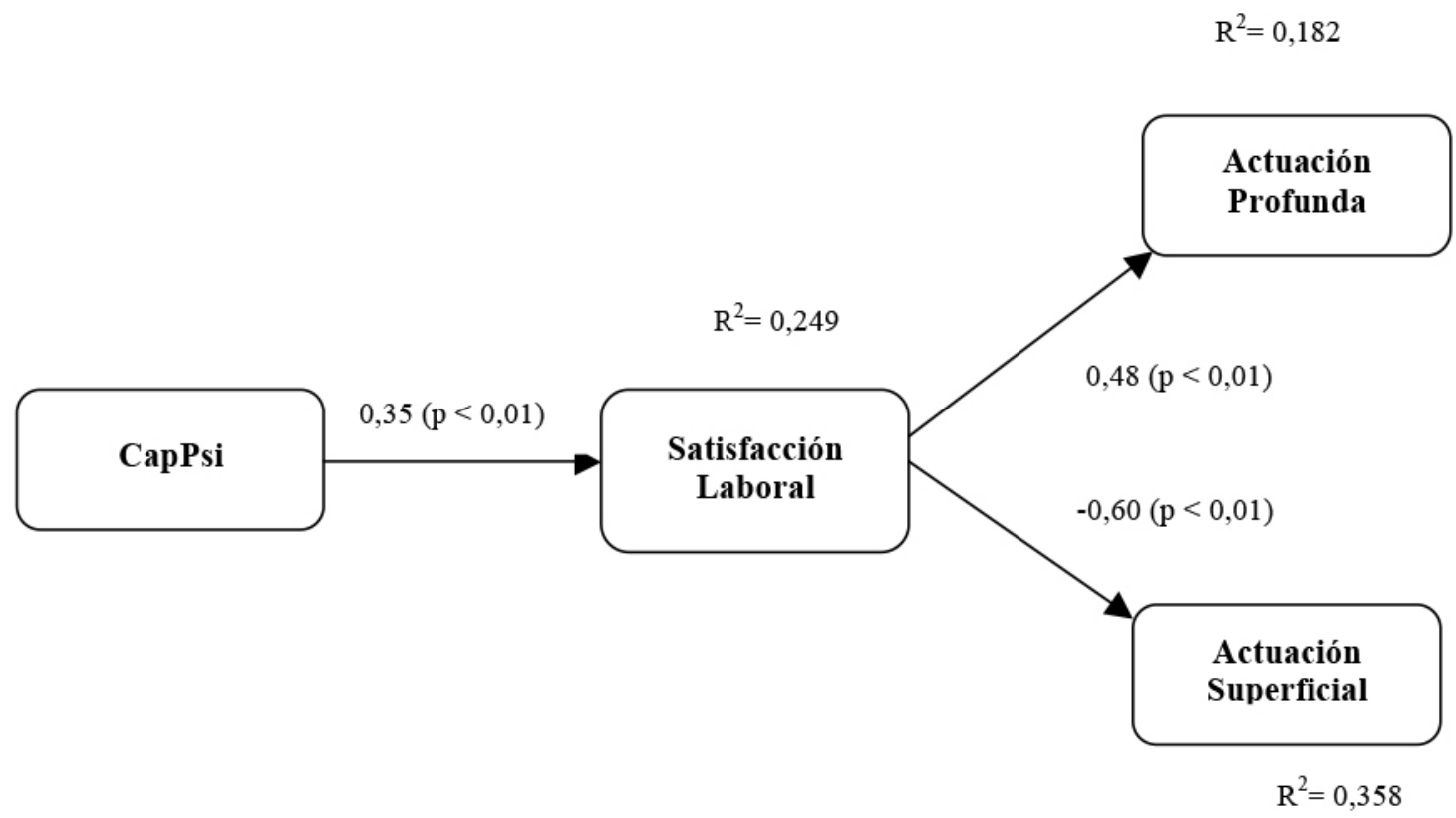

Figura 1. Coeficientes Estandarizados y Proporción de Varianza Explicada Correspondiente al Modelo de Mediación Total.

\section{Discusión}

El presente trabajo se propuso explorar si la satisfacción laboral desempeña el rol de mediador entre los recursos psicológicos y las estrategias de regulación emocional implementadas por los trabajadores. Basándose en la metodología de modelos rivales (Bentler, 2006), el estudio contrastó dos modelos explicativos en los que la satisfacción laboral asumió los roles de mediador total y mediador parcial, respectivamente. La verificación empírica realizada permite arribar a una conclusión sobre cuál es el modelo más idóneo. En este sentido, los hallazgos del presente estudio indican que el modelo de mediación total constituye, para la muestra bajo estudio, una propuesta explicativa superadora frente a un modelo alternativo de mediación parcial. De acuerdo a los resultados obtenidos es posible aseverar que la satisfacción actúa como mecanismo explicativo subyacente a las relaciones entre el CapPsi y las estrategias de regulación emocional siendo, precisamente, la satisfacción (o insatisfacción) la principal promotora de actuaciones profundas o superficiales, respectivamente.

Un análisis pormenorizado del modelo muestra que el CapPsi se relaciona significativamente con la satisfacción de los trabajadores; hallazgo que coincide con los informados por investigaciones recientes (Alessandri et al., 2015; Avey et al., 2011; Badran \& Youssef-Morgan, 2015; Salessi \& Omar, 2016b). En conjunto, estos resultados indican que las personas con alto CapPsi esperan que les sucedan cosas buenas en el trabajo; creen que son artífices de su propio éxito y están mejor predispuestas para sortear los reveses y contratiempos laborales. Su elevada motivación explica que sean estos trabajadores quienes, precisamente, desarrollen emociones positivas que, a su vez, retroalimentan sus recursos psicológicos y generan espirales de positividad creciente.

En lo que hace a las vinculaciones entre la satisfacción y las estrategias de regulación, hasta el momento de la presente investigación no se contaba con evidencias científicas que demostraran que la primera puede influir significativamente sobre las segundas. Por lo que, este estudio ha avanzado un paso importante en esa dirección. De acuerdo a los resultados obtenidos la actuación superficial parece estar relacionada a la insatisfacción de los trabajadores, mientras que la actuación profunda sería favorecida por actitudes laborales positivas. La magnitud de los coeficientes obtenidos en los análisis estructurales indica que las relaciones entre satisfacción y regulación emocional son fuertes y significativas superando ampliamente a las relaciones observadas entre CapPsi y regulación. Lejos de ser problemático, dicho hallazgo se muestra en línea con lo comunicado por otros autores (Begenirbaş, 2015; Hur et al., 2016; Uğurlu-Kara \& Begenirbaş, 2016), quienes también han informado relaciones modestas 
entre CapPsi, actuación profunda y actuación superficial. Frente a este panorama, conjeturar un posible rol mediador de la satisfacción parece ser un interés científico legítimo.

Al analizar holísticamente las relaciones entre las variables bajo estudio surge que los recursos psicológicos tuvieron efectos indirectos significativos sobre la regulación a través de la satisfacción laboral. Sin embargo, no se registraron efectos directos residuales por parte del CapPsi. Antes estos hallazgos es posible afirmar que el modelo de mediación total es la explicación más idónea para analizar el interjuego entre estas variables. Sin embargo, es menester advertir que los resultados obtenidos son sólo válidos para la muestra seleccionada. Aún frente a la necesidad de refinamientos ulteriores, los resultados alcanzados son promisorios, desde el momento que sindican a la satisfacción laboral como un engranaje fundamental. En efecto, considerando que los mayores coeficientes de regresión se observan, precisamente, en el sendero comprendido entre la satisfacción y la regulación, es factible precisar que el mismo constituye un tramo crucial para comprender acabadamente de qué modo el CapPsi influye sobre el comportamiento de los empleados. En línea con los postulados del modelo de ampliación y construcción emocional (Fredrickson, 2001), resulta factible asumir que el estado motivacional y positivo asociado a un alto CapPsi facilitaría una mayor satisfacción laboral; al tiempo que el componente afectivo y placentero que la satisfacción supone propiciaría cambios cognitivos que posibilitarían llevar a cabo actuaciones profundas. De esta manera, por ejemplo, estrategias como la atención selectiva y la reevaluación cognitiva se verían promovidas por la mayor apertura mental y el estado expansivo que propician la afectividad positiva (Grandey et al., 2013; Gross, 2010). En contraposición, en condiciones en las que el CapPsi sea escaso, sería esperable que los trabajadores sean más vulnerables a experimentar ansiedad, dudas, menor confianza y menor autoestima. La evaluación de las circunstancias laborales podría estar sesgada por el pesimismo y la falta de motivación, siendo inminente el desarrollo de actitudes negativas hacia el trabajo y hacia la organización. Una vez instaurada la insatisfacción, la emocionalidad negativa asociada sería responsable de limitar las posibilidades de los trabajadores para regular las emociones, Ilevándolos a actuar superficialmente. Así, por ejemplo, en lugar de intentar reinterpretar desde una perspectiva diferente su situación, quedarían confinados a rumiaciones y quejas que sólo lograrían incrementar su insatisfacción. Ante la dificultad de afrontar constructivamente su propia afectividad negativa, los trabajadores insatisfechos optarían por enmascarar su estado de ánimo (por ejemplo, con una expresión neutral), inhibir todo tipo de manifestación emocional o, en el mejor de los casos, reprimir su malestar ocultándolo tras una sonrisa vacía. En última instancia, la actuación superficial podría funcionar como una estrategia para hacer frente a la desmoralización y la escasa realización personal asociados a la menor satisfacción con el trabajo.

\section{Implicancias Prácticas, Fortalezas y Limitaciones}

Los resultados del estudio realizado aportan un conjunto de implicancias prácticas para las organizaciones. Considerando los esfuerzos de toda empresa de servicios por lograr que sus empleados traten con amabilidad y cortesía a sus clientes, que eviten las confrontaciones verbales y que intenten solucionar los problemas armoniosamente, gerentes y encargados de recursos humanos podrían introducir prácticas organizacionales de bajo costo con miras a reducir las actuaciones superficiales e incrementar las actuaciones profundas. En términos generales, se trataría de prácticas que contemplen instancias de entrenamiento para mejorar la regulación emocional; la creación de espacios de empoderamiento de los trabajadores en los procesos de resolución de problemas durante la atención al público; el fortalecimiento del autocontrol afectivo frente a situaciones potencialmente desestabilizadoras, y similares. Los resultados podrían capitalizare, a su vez, para propiciar ambientes laborales que contribuyan al florecimiento del capital psicológico, especialmente en las actividades vinculadas con el rubro servicios. Sobre todo porque desde la irrupción de este constructo en el escenario de la psicología organizacional positiva (Luthans et al., 2007), más que un rasgo fijo, ha sido considerado un estado susceptible de cambio y desarrollo. Por lo que, contar con empleados autoeficaces, resilientes, optimistas y esperanzados (vale decir, con alto CapPsi), favorecería la satisfacción en el trabajo. Consecuentemente, la mayor satisfacción laboral podría garantizar relaciones interpersonales signadas por la autenticidad y la empatía; aspectos que, en última instancia, promoverían resultados positivos tanto para la organización como para los propios empleados. En este sentido, la organización se vería recompensada con desempeños de mayor calidad y clientes más leales; en tanto que los empleados experimentarían mayores niveles de bienestar y salud ocupacional. 
Como todo trabajo empírico, el presente entraña fortalezas y debilidades. Entre sus limitaciones hay que remarcar, en primer lugar, el diseño transversal utilizado, el que limita cualquier inferencia de causalidad en sentido estricto entre las variables estudiadas. Por lo que, a efectos de establecer verdaderamente el orden causal de las relaciones encontradas en el presente trabajo, sería muy valioso que futuras investigaciones empleen diseños longitudinales. En segundo lugar, puesto que el procedimiento de selección muestral no ha sido probabilístico, la generalización de los resultados a la población es desaconsejada. No obstante, cabe aclarar que con el propósito de minimizar cualquier inconveniente derivado del muestreo se procuró, por un lado, que la muestra ascendería a un tamaño suficiente como para detectar relaciones estadísticamente significativas. Por otro lado, dentro de los límites impuestos por los criterios de exclusión, la composición final de la muestra incluyera empresas de diferentes sectores organizacionales y niveles de complejidad. Finalmente, una tercera limitación del estudio podría estar referida a la posible contaminación de las respuestas por los efectos de la deseabilidad social (vale decir, la tendencia a mostrar una imagen mejorada de uno mismo). Posiblemente, la mayor tendencia a la deseabilidad social, aun cuando los participantes tuvieron garantizado el anonimato durante el proceso de recolección de datos, podría haberse activado frente a los ítems que evalúan la actuación superficial ya que suponen patrones de comportamiento deliberadamente inauténticos. Para superar esta limitación, en futuros estudios se podría incluir alguna de las tantas medidas de deseabilidad social disponibles en la batería de recolección de datos.

Entre las fortalezas de esta investigación, es conveniente destacar que constituye una contribución genuina al conocimiento de los antecedentes de la regulación emocional en el trabajo. Posiblemente, el mayor aporte que pueda atribuírsele a este estudio sea proponer una perspectiva novedosa como alternativa para comprender el binomio satisfacción laboral-regulación emocional; así como, el haber demostrado que, además de ser una variable típicamente de resultado, la satisfacción también puede jugar un rol protagónico como variable mediadora. Al incluir en un mismo modelo explicativo un antecedente distal como el CapPsi y uno proximal como las experiencias de satisfacción-insatisfacción laboral, este trabajo proporciona evidencia preliminar sobre los mecanismos que se activan tras las sonrisas fingidas y las expresiones auténticas de las que los trabajadores son protagonistas.
Como corolario del trabajo realizado futuros estudios pueden continuar ampliando el mapa de predictores del trabajo emocional. En este sentido, considerando, por ejemplo, que el porcentaje de varianza explicada de la actuación profunda resultó comparativamente menor que el correspondiente a la actuación superficial, sería provechoso investigar otros antecedentes. Dada la naturaleza de los constructos involucrados, es posible que variables tales como el compromiso organizacional, la confianza organizacional y la pasión por el trabajo emerjan como antecedentes idóneos de las actuaciones profundas y superficiales. La exploración de estos caminos alternativos estaría marcando la agenda para las próximas investigaciones en el área.

\section{Referencias}

Alessandri, G., Borgogni, L., Consiglio, C., \& Mitidieri, G. (2015). Psychometric properties of the Italian version of the Psychological Capital Questionnaire. International Journal of Selection and Assessment, 23(2), 149-159. doi: 10.1111/ijsa.12103

Ato, M., López, J., \& Benavente, A. (2013). Un sistema de clasificación de los diseños de investigación en psicología. Anales de Psicología, 29(3), 1038-1059. doi: 10.6018/analesps.29.3.178511

Avey, J. B., Reichard, R. J., Luthans, F., \& Mhatre, K. H. (2011). Metaanalysis of the impact of positive psychological capital on employee attitudes, behaviours and performance. Human Resource Development Quarterly, 22(2), 127-152. doi: 10.1002/hrdq.20070.

Baron, R. M., \& Kenny, D. A. (1986). The moderator-mediator variable distinction in social psychological research: conceptual, strategic and statistical considerations. Journal of Personality and Social Psychology, 51, 1173-1182. doi: 10.1037/0022-3514.51.6.1173

Badran, M. A., \& Youssef-Morgan, C. M. (2015). Psychological capital and job satisfaction in Egypt. Journal of Managerial Psychology, 30(3), 354-370. doi: 10.1108/JMP-06-2013-0176.

Begenirbaş, M. (2015). The effects of psychological capital on employees' emotional displays and work alienation: a research in the health sector. Suleyman Demirel University Journal of Faculty of Economics \& Administrative Sciences, 20(3), 249-263.

Bentler, P. (2006). EQS 6 Structural Equations Program manual. Encino: Multivariate Software.

Fornell, C., \& Larcker, D.F. (1981). Evaluating structural equation models with unobservable variables and measurement error. Journal of Marketing Research, 18(1), 39-50.

Fredrickson, B. (2001). The role of positive emotions in positive psychology: the broaden-and-build theory of positive emotions. American Psychologist, 56(3), 218-226. doi: 10.1037/0003-066X.56.3.218.

Grandey, A. (2015). Smiling for a wage: what emotional labor teaches us about emotion regulation. Psychological Inquiry, 26(1), 54-60. doi: 10.1080/1047840X.2015.962444

Grandey, A., Diefendorff, J., \& Rupp, D. (2013). Emotional labor in the 21st century: diverse perspectives on emotion regulation at work. Nova lorque: Psychology Press/Routledge. doi: $10.4324 / 9780203100851$ 
Capital psicológico y regulación emocional en el trabajo: El rol mediador de la satisfacción laboral

Gross, J. J. (2010). Emotion regulation. In M. Lewis, J. M. HavilandJones, \& L. Feldman Barrett (Eds.), Handbook of emotions (pp. 497-512). Nova lorque: Guilford Press.

Hobfoll, S. E. (2011). Conservation of resource caravans, and engaged settings. Journal of Occupational and Organizational Psychology, 84, 116-122. doi: 10.1111/j.2044-8325.2010.02016.x.

Hochschild, A. R. (1983). The managed heart: commercialization of human feeling. Berkley: University of California press. doi: 10.1093/ $\mathrm{sf} / 64.1 .223$

Hopwood, C. (2007). Moderation and mediation in structural equation modeling: applications for early intervention research. Journal of Early Intervention, 29, 262-272. doi: 10.1177/105381510702900305

Hur, W., Rhee, S., \& Ahn, K. (2016). Positive psychological capital and emotional labor in Korea: the job demands-resources approach. International Journal of Human Resource Management, 27(5), 477500. doi: 10.1080/09585192.2015.1020445

Jiang, X., Jiang, Z., \& Park, D. (2013). Emotional labor strategy and job satisfaction: a Chinese perspective. Social Behavior \& Personality: An International Journal. 41, 933-938. doi: 10.2224/ sbp.2013.41.6.933.

Judge, T., \& Kammeyer-Mueller, J. (2012). Job attitudes. Annual Review of Psychology, 63, 341-367. doi: 10.1146/ annurev-psych-120710-100511

Liu, Y., Prati, L., Perrewe, P., \& Brymer, R. (2010). Individual differences in emotion regulation, emotional experiences at work, and work-related outcomes: a two-study investigation. Journal of Applied Social Psychology, 40(6), 1515-1538. doi: 10.1111/j.1559-1816.2010.00627.x

Luthans, F., Youssef, C., \& Avolio, B. J. (2007). Psychological capital: developing the human competitive edge. Oxford: Oxford University Press.

Mao, J., \& Tang, C. (2015). Team psychological capital and teachers organizational citizenship behavior: The mediating role of job satisfaction. Chinese Journal of Clinical Psychology, 23(4), 736740. Recuperado de http://en.cnki.com.cn/Article_en/CJFDTotalZLCY201504040.htm

Omar, A. (2015). Constructos fundacionales de la Psicología Organizacional Positiva.
PsyCap. Revista Latinoamericana de Psicología Positiva, 2, 72-87. Recuperado de http://psycap.cl/wp-content/uploads/2016/01/ Revista_Psycap0215.pdf

Paris, L., \& Omar, A. (2008). Predictores de satisfacción laboral en médicos y enfermeros. Estudos de Psicologia, 13(3), 233-244. doi: 10.1590/S1413-294X2008000300006

Podsakoff, P., MacKenzie, S., \& Podsakoff, N. (2012). Sources of method bias in social science research and recommendations on how to control it. Annual Review of Psychology, 65, 539-569. doi: 10.1146/annurev-psych-120710-100452

Salessi, S. (2012). Demandas emocionales asociadas al rol laboral. Consideraciones conceptuales. Acta Psiquiátrica y Psicológica de América Latina, 58(1), 48-52. Recuperado de http://www.acta.org. ar/04-WebForms/frmResumen.aspx? IdArticulo=22\&Abonado $=$

Salessi, S. (2014). Satisfacción laboral: acerca de su conceptualización, medición y estado actual del arte. Revista de Psicología, 19(10), 67-83. Recuperado de http://bibliotecadigital.uca.edu.ar/ repositorio/revistas/psicologia19.pdf

Salessi, S., \& Omar, A. (2016a). Desarrollo y validación de una nueva escala para medir actuación emocional en el trabajo. Revista Iberoamericana de Diagnóstico y Evaluación Psicológica, 41(1), 66-79. Recuperado de http://www.aidep.org/03_ridep/R41/Art6.pdf

Salessi, S., \& Omar, A. (2016b). Satisfacción laboral genérica. Propiedades psicométricas de una escala para medirla. Alternativas en Psicología, 34, 93-108. Recuperado de http://www.alternativas. me/numeros/23-numero-34-febrero-julio-2016/116-satisfaccion-laboral-generica-propiedades-psicometricas-de-una-escala-para-medirla

Tabachnick, B. G., \& Fidell, L. S (2013). Using multivariate statistics (6 $6^{\text {ta }}$ Ed.). Boston, MA: Pearson.

Uğurlu Kara, A., \& Begenirbaş, M. (2016). Do psychological capital perceptions of employees affect their emotional role behaviors? A research in tourism sector. EUL Journal of Social Sciences, 7(1), 50-66.

Wei, W., Guo, L., Ye, M., Liao, G., \& Yang, Z. (2016). Work-family conflict and safety participation of high-speed railway drivers: Job satisfaction as a mediator. Accident Analysis and Prevention, 95, 97-103. doi: 10.1016/j.aap.2016.06.022

Solana Salessi, Doctora en Psicología por Universidad Nacional de La Plata (UNLP), Argentina, Becaria Postdoctoral del Consejo Nacional de Investigaciones Científicas y Técnicas (CONICET), Argentina, es Profesora

Asociada de la Universidad Nacional de Rafaela (UNRaf), Argentina, Profesora Adjunta de la Facultad de Derecho y Ciencias Sociales del Rosario, Pontificia Universidad Católica Argentina (UCA).

Dirección: Balcarce 855 - 9 A (2000) - Rosario, Santa Fe, Argentina. Teléfono: +54-03404-15500180. Correo electrónico: solanasalessi@gmail.com

Alicia Omar, Doctora en Psicología por la Universidad Nacional de Cuyo (UNCUYO), Argentina, es Investigadora Independiente del Consejo Nacional de Investigaciones Científicas y Técnicas (CONICET), Argentina. Correo electrónico: agromar@yahoo.com

Recebido em 12.Jun.16 Revisado em 10.Jan.17 Aceito em 06.Mar.17 